\title{
Avaliação do estresse ocupacional em agentes comunitários de saúde de uma estratégia saúde da família
}

\author{
Evaluation of occupational stress in healthcommunity \\ agents of a family health strategy
}

Cleverton Chaves dos Reis ${ }^{1}$, Sérgio Alexandre Oliveira Malcher ${ }^{1}$

Resumo Objetivo: Determinar a prevalência dos casos de estresse ocupacional em agentes comunitários de saúde lotados na USF águas brancas localizada no município de Ananindeua-PA. Método: Um estudo quantitativo, observacional, transversal e descritivo durante o período de fevereiro a outubro de 2017. Constituída pelo total de 20 agentes comunitários de saúde (ACS) que estão devidamente lotados e trabalhando regularmente nas 3 estratégias saúde da família que compõe a UESF das Águas Brancas. Sendo 7 ACS na ESF águas brancas I, 7 ACS na ESF águas brancas II e 6 ACS na ESF Dom Bosco. Resultados: O perfil sócio demográfico foram encontrados $70 \%$ do sexo feminino, faixa etária de 50-59 anos com 35\%, raça 65\% são pardos, estado civil $50 \%$ são casados, com escolaridade 35\% tem o ensino médio completo; Em relação aos fatores preceptores do estresse no trabalho a partir do inquérito ocupacional temos $70 \%$ que classificaram como média a satisfação com o trabalho, $75 \%$ consideram baixo o conforto no ambiente de trabalho, $40 \%$ consideram a temperatura é que mais causa incomoda quanto as condições do ambiente, $70 \%$ afirmaram que as tarefas não são monótonas já que estão intensamente executando seu trabalho, 40\% afirmaram que o volume de serviço é excessivo sazonal, $40 \%$ são estudantes e $25 \%$ realizam trabalhos extras e $15 \%$ sofreram acidente de trabalho. Todos trabalham $8 \mathrm{~h}$ durante a semana e $25 \%$ trabalham nos fim de semana na informalidade. Em relação aos sinais e sintomas mais prevalentes estão fadiga, dor nos músculos pescoço e ombro com 85\% dos casos seguidas de dor de cabeça, indigestão e dor lombar com $80 \%$. Em relação ao estresse ocupacional tivemos $45 \%$ para faixa moderada e o mesmo valor percentual para estresse intenso totalizando $90 \%$ dos casos. Considerações finais: Essa situação serve de alerta para que medidas corretivas e preventivas possam ser tomadas a fim de evitarmos a queda de desempenho e o adoecimento desses trabalhadores.

Descritores: estresse ocupacional; agentes comunitários de saúde; estratégia saúde da família.

Summary Purpose: Determine the prevalence of occupational stress in health Community agentes crowded at USF Águas Brancas in Ananindeua-PA. Method: It will be a quantitative, observational, transversal and descriptive study during the period from February to October 2017. They were made up of a total of 20 community health agents (ACS) who are adequately crowded and working regularly in the 3 health strategies of the family that make up the UESF of White Waters. Being 7 ACS in the ESF white waters I, 7 ACS in the ESF white waters II and 6 ACS in the ESF Don Bosco. Results: The sociodemographic profile has 70\% female, $50-59$ age group with $35 \%$, race $65 \%$ are brown, civil status $50 \%$ are married, with education $35 \%$ have completed high school; Regarding occupational stressors, $70 \%$ rated occupational satisfaction as the average, $75 \%$ consider comfort in the work environment to be low, $40 \%$ considered that temperature is what causes more discomfort. $70 \%$ stated that the tasks are not monotonous since they are intensely performing their work, $40 \%$ stated that the volume of service is excessive seasonal, $40 \%$ are students and $25 \%$ work extra and $15 \%$ have suffered an accident at work. Everybody works 8 AM during the week and $25 \%$ work weekends on the informality. In relation to the most prevalent signs and symptoms are fatigue, pain in the neck and shoulder muscles with $85 \%$ of cases followed by headache, indigestion and low back pain with $80 \%$. Regarding occupational stress we had $45 \%$ for moderate range and the same percentage value for intense stress totaling $90 \%$ of the cases. Final considerations: This situation serves as an alert for corrective and preventive measures to be taken in order to avoid the fall in performance and the sickness of these workers Keywords: occupational stress; community health workers; family health strategy.

'Universidade do Estado do Pará - UEPA, Belém, PA, Brasil

Fonte de financiamento: Nenhuma.

Conflito de interesses: Os autores declaram não haver conflitos de interesse.

Recebido: Abril 06, 2018

Aceito: Maio 05, 2018

Trabalho realizado na Unidade Estratégia Saúde da Família das Águas Brancas, Ananindeua, PA, Brasil.

Copyright Reis et al. Este é um artigo publicado em acesso aberto (Open Access) sob a licença Creative Commons Attribution, que permite uso, distribuição e reprodução em qualquer meio, sem restrições desde que o trabalho original seja corretamente citado. 


\section{Introdução}

A palavra estresse foi usada pela primeira vez em meados do século passado para definir o processo de reação do organismo a uma situação de perigo. Os fatores estressores impulsionam a nossa luta pela vida, pois recebemos estímulos 24 horas por dia. Alguns estímulos são conscientizados, outros, captados apenas pelo nosso subconsciente; alguns são agradáveis, outros não; alguns, em primeira instância, insignificantes, mas, pela constância da repetição, tornam-se patogênicos ${ }^{1}$. Nesse sentido, o estresse ocupacional provoca o aparecimento de doenças que determinam o absenteísmo, causando prejuízo tanto para o funcionário quanto para o empregador.

O processo de estresse é resultado de pressões emocionais repetitivas presentes no ambiente de trabalho. Está associada a ocupações assistenciais, as quais têm contato direto com usuários do serviço, como profissionais da educação e da saúde ${ }^{2}$. Isso ocorre nesses profissionais, dentre outros motivos, devido à divergência entre a expectativa do profissional e a realidade que este encontra no trabalho ${ }^{3}$. Entender quais fatores interferem na saúde do trabalhador e, consequentemente, na sua qualidade de vida é de grande importância para que se possa criar estratégias capazes de atuar diretamente nessas situações do trabalho que geram sofrimento e agravos à saúde ${ }^{4}$.

Em 1991, nasceu o Programa de Agentes Comunitários de Saúde (PACS) com o intuito de melhorar a qualidade de vida da população, com investimento na educação em saúde, tendo como personagem fundamental os agentes comunitários de saúde (ACS) $)^{5,6}$

A equipe e a comunidade depositam grandes anseios e expectativas nos ACS, os quais podem vir a se tornar fortes estressores para a saúde desses profissionais ${ }^{3}$. Outras dificuldades encontradas por esse profissional são: quantidade elevada de pessoas da comunidade atendida, associada à falta de entendimento da população quanto ao seu trabalho; dificuldade de resolução dos problemas da comunidade, que depende do envolvimento de toda equipe multidisciplinar; falta de organização do serviço e relações conflituosas na equipe.

Neste contexto e por não termos pesquisas e/ou fontes bibliográficas atualizadas sobre o tema em questão, realizou-se o presente estudo, cujo objetivo foi investigar os casos de estresse ocupacional em ACS lotados em unidade de saúde da família no município de Ananindeua (PA).

\section{Método}

O estudo caracterizou-se se como quantitativo, observacional, transversal e descritivo durante o período de fevereiro a outubro de 2017.Constituído pelo total de 20 agentes comunitários de saúde (ACS) que estão devidamente lotados e trabalhando regularmente nas 3 estratégias saúde da família que compõe a UESF das Águas Brancas e ainda, com mais de 3 anos de tempo de serviço. Sendo 7 ACS na ESF águas brancas I, 7 ACS na ESF águas brancas II e 6 ACS na ESF Dom Bosco. Foram realizados 3 encontros em 3 terças-feiras consecutivas com os ACS de suas respectivas equipes sendo divididas da seguinte maneira: ESF águas brancas 1, águas brancas 2 e Dom Bosco. Esse dia da semana foi escolhido, terça-feira, como também o horário de expediente, das $9 \mathrm{~h}$ às $11 \mathrm{~h}$ da manhã, visto que ocorrem as atividades da educação em saúde.

Foi explicada a finalidade da pesquisa e posteriormente submetidos a entrevista com utilização de protocolo de pesquisa constituído por perguntas objetivas relacionados ao estresse ocupacional baseado no teste de detecção do estresse com os trabalhadores da saúde, feito por Baccaro ${ }^{7}$. Além disso, foram feitas perguntas sobre o perfil epidemiológico, inquérito ocupacional e aplicado o TCLE que foi assinado pelos sujeitos da pesquisa.

Os critérios para inclusão na pesquisa foram de ACS que estão devidamente lotados e trabalhando regularmente na UESF das Águas Brancas com mais de 3 anos de tempo de serviço. Foram excluídos todos aqueles que não corresponderam ao perfil citado anteriormente como também aqueles que se recusaram a participar da pesquisa.

Os dados obtidos foram representados em gráficos e/ou tabelas, e posteriormente, foram submetidos à análise estatística descritiva com programa Bioest $5.3^{\circledR}$, de acordo com a natureza das variáveis. Para agrupamento de dados e confecções de gráficos e tabelas foi utilizado o software Excel $2016{ }^{\circledR}$, sendo informados os valores em números absolutos e relativos.

Todos os sujeitos da presente pesquisa foram estudados segundo os preceitos da Declaração de Helsinque e do Código de Nuremberg, respeitadas as normas de pesquisa envolvendo seres humanos (Res. CNS 466/12), com aprovação do projeto pelo Comitê de Ética em Pesquisa (CEP) em seres humanos do Centro de Ciências Biológicas e da Saúde da Universidade do Estado do Pará (CCBS/UEPA) por intermédio de parecer consubstanciado $\mathrm{n}^{\circ}$ 2.339.368 e assinatura do termo de Consentimento Livre e Esclarecido (TCLE) pelos sujeitos da pesquisa. 


\section{Resultados}

\section{Discussão}

O perfil sócio demográfico do estudo (Tabela 1) é de 70\% do sexo feminino, faixa etária de 5059 anos com $35 \%$, seguida da faixa etária de 30-39 anos (30\%), raça em 65\% são pardos seguido de negros 25\%, Já o estado civil, $50 \%$ são casados seguidos $35 \%$ de solteiros, com escolaridade $35 \%$ tem o ensino médio completo e $30 \%$ com ensino médio incompleto em concordância e contrapontos do estudos de Santos et al $^{8}$ onde na presente estudo, a maioria dos profissionais é do gênero feminino; 34,2\% apresentaram idade entre 18 e 29 anos, 39,5\%, entre 30 a 39 anos, 18,4\%, entre 40 a 49 anos e 7,9\% com cinquenta anos ou mais. Constatou-se que 5,3\% dos agentes possuem o ensino fundamental incompleto; $65,8 \%$, o ensino médio completo; 7,9\%, ensino médio incompleto e 21,1\%, o ensino superior incompleto. Entre as duas pesquisas, identificou-se respectivamente que $35 \%$ e $65,8 \%$ dos agentes possuíam ensino médio sendo uma informação significativa visto que provavelmente define essa situação é a pequena oferta de empregos e os pequenos salários pagos. Outra casuística reafirma as duas pesquisas demonstrando resultados similares já que a maioria dos ACS pesquisados eram do sexo feminino (87,8\%), na faixa etária entre 30 a 49 anos $(68,9 \%)$, de raça / cor parda (64\%) e escolaridade possuem $2^{\circ}$ grau completo (58,5\%). Dentre os profissionais pesquisados, $48,2 \%$ são casados ou tem união estável ${ }^{9}$.

Tabela 1. Perfil Sociodemográfico sobre estresse ocupacional dos ACS da USF Águas Brancas, Ananindeua-PA

\begin{tabular}{|c|c|c|}
\hline VARIÁVEL & TOTAL & $\%$ \\
\hline \multicolumn{3}{|l|}{ SEXO } \\
\hline MASCULINO & 6 & 30 \\
\hline FEMININO & 14 & 70 \\
\hline \multicolumn{3}{|l|}{ FAIXA ETÁRIA } \\
\hline 20-29 ANOS & 3 & 15 \\
\hline 30-39 ANOS & 6 & 30 \\
\hline 40-49 ANOS & 3 & 15 \\
\hline 50-59 ANOS & 7 & 35 \\
\hline 60 OU MAIS & 1 & 5 \\
\hline \multicolumn{3}{|l|}{ RAÇA } \\
\hline NEGROS & 5 & 25 \\
\hline BRANCOS & 2 & 10 \\
\hline PARDOS & 13 & 65 \\
\hline AMARELOS & 0 & 0 \\
\hline \multicolumn{3}{|l|}{ ESTADO CIVIL } \\
\hline SOLTEIRO & 7 & 35 \\
\hline CASADOS & 10 & 50 \\
\hline UNIÃO ESTÁVEL & 3 & 15 \\
\hline \multicolumn{3}{|l|}{ ESCOLARIDADE } \\
\hline EFI & 0 & 0 \\
\hline EFC & 0 & 0 \\
\hline EMI & 6 & 30 \\
\hline EMC & 7 & 35 \\
\hline ESI & 4 & 20 \\
\hline ESC & 3 & 15 \\
\hline
\end{tabular}

Legenda: $\mathrm{EFI}$ = Ensino fundamental incompleto; $\mathrm{EFC}=$ Ensino fundamental completo; EMI = Ensino Médio Incompleto; EMC = Ensino médio completo; ESI = Ensino Superior Incompleto; ESC = Ensino Superior Completo. Fonte: Protocolo de pesquisa. 
Em relação aos fatores preceptores do estresse no trabalho (Tabela 2) a partir do inquérito ocupacional temos $70 \%$ que classificaram como média a satisfação com o trabalho, $75 \%$ consideram baixo o conforto no ambiente de trabalho, $40 \%$ consideraram a temperatura é que mais causa incomoda quanto as condições do ambiente, $70 \%$, afirmaram que as tarefas não são monótonas já que estão intensamente executando seu

Tabela 2. Fatores preceptores do estresse ocupacional dos ACS da USF Águas Brancas, Ananindeua-PA

\begin{tabular}{|c|c|c|}
\hline INQUÉRITO OCUPACIONAL & TOTAL & $\%$ \\
\hline \multicolumn{3}{|l|}{ GRAU DE SATISFAÇÃO NO TRABALHO } \\
\hline ALTO & 1 & 5 \\
\hline MÉDIO & 14 & 70 \\
\hline BAIXO & 5 & 25 \\
\hline \multicolumn{3}{|l|}{ CONFORTO NO AMBIENTE DE TRABALHO } \\
\hline ALTO & 0 & 0 \\
\hline MEDIO & 5 & 25 \\
\hline BAIXO & 15 & 75 \\
\hline \multicolumn{3}{|l|}{ CONDIÇÕES AMBIENTAIS } \\
\hline NENHUM & 5 & 25 \\
\hline MOBILIÁRIO & 4 & 20 \\
\hline RUÍDO & 1 & 5 \\
\hline TEMPERATURA & 8 & 40 \\
\hline ILUMINAÇÃO & 2 & 10 \\
\hline \multicolumn{3}{|l|}{ MONOTONIA DE TAREFAS } \\
\hline SIM & 6 & 30 \\
\hline NÃO & 14 & 70 \\
\hline \multicolumn{3}{|l|}{ VOLUME DE SERVIÇO } \\
\hline ADEQUADO & 8 & 40 \\
\hline EXCESSIVO SAZONAL & 8 & 40 \\
\hline EXCESSIVO & 4 & 20 \\
\hline \multicolumn{3}{|l|}{ ESTUDANTE } \\
\hline SIM & 8 & 40 \\
\hline NÃO & 12 & 60 \\
\hline \multicolumn{3}{|l|}{ ATIVIDADE EXTRA } \\
\hline NÃO & 7 & 35 \\
\hline TRABALHO & 5 & 25 \\
\hline ESTUDO & 8 & 40 \\
\hline \multicolumn{3}{|l|}{ ACIDENTE DE TRABALHO } \\
\hline SIM & 3 & 15 \\
\hline NÃO & 17 & 85 \\
\hline \multicolumn{3}{|c|}{ JORNADA DE TRABALHO DURANTE A SEMANA } \\
\hline $8 \mathrm{H}$ & 20 & 100 \\
\hline MAIS DE 8H & 0 & 0 \\
\hline \multicolumn{3}{|l|}{ TRABALHO AOS FINS DE SEMANA } \\
\hline SIM & 5 & 25 \\
\hline NÃO & 15 & 75 \\
\hline
\end{tabular}

Fonte: Protocolo de pesquisa. 
trabalho, 40\% afirmaram que o volume de serviço é excessivo sazonal, quanto a lateralidade 70\% são destros, $50 \%$ trabalham alternando a sua vontade como também em pé, $40 \%$ são estudantes e $25 \%$ realizam trabalhos extras. $15 \%$ sofreram acidente de trabalho, todos trabalham 8 h durante a semana e $25 \%$ trabalham nos fim de semana na informalidade. Não há trabalhos com as mesmas características do inquérito ocupacional utilizado, apenas trata sobre jornada excessiva de trabalho. Em contrapartida, outro estudo mostrou uma correlação positiva entre realização pessoal, satisfação com a organização hierárquica do trabalho e satisfação com o ambiente físico do trabalho, assim como oportunidade de crescimento profissional ${ }^{10}$.

Em relação aos sinais e sintomas mais prevalentes (Tabela 3) estão fadiga, dor nos músculos pescoço e ombro com $85 \%$ dos casos seguidas de dor de cabeça indigestão e dor lombar com $80 \%$ o que está de acordo com o estudo de Mota et al..$^{9}$ onde os profissionais estudados, $57,7 \%$ apresentam grau moderado ou grave de exaustão emocional; $51,8 \%$ apresentam grau moderado ou grave de despersonalização e $59 \%$ de moderado a alto envolvimento pessoal no trabalho. Em se tratando da casuística de $\mathrm{Lima}^{11}$, a sintomatologia do estresse mais percebida pelos trabalhadores de saúde caracterizou-se por $81,25 \%$ de dor muscular e ansiedade; $62,5 \%$ dor de cabeça e tensão muscular; $56,25 \%$ sensação de pressão no peito; $50 \%$ gastrite; $43,75 \%$ azia, problemas do aparelho urinário, DORT e insônia; 37,5\% má digestão, palpitação e irritabilidade; $31,25 \%$ erupção cutânea e diminuição da atividade imunológica e dificuldade de concentração seguido de $25 \%$ de problemas respiratórios, isolamento social e depressão. Os dois estudos mostraram poucas distorções e ratificaram o predomínio de alguns sinais e sintomas. Frequentemente, o trabalho da equipe de Saúde da Família e especialmente dos Agentes Comunitários de Saúde exige a necessidade de se produzir muito em pouco tempo, bem como a repetição de tarefas. Segundo Glina ${ }^{12}$, os trabalhadores recebem sobrecargas tanto qualitativas, quanto quantitativas, representando alto volume de trabalho mental por unidade de tempo e complexidade do trabalho frente a capacidades e experiências do trabalhador. Essas sobrecargas representam fatores estressantes em um ambiente ocupacional representando uma preocupante situação, potencialmente causadora de doenças nos trabalhadores, já que a combinação entre altas demandas e baixos controles sobre o trabalho é sabidamente situação de estresse ocupacional.

Em relação ao estresse ocupacional (Tabela 4) tivemos $45 \%$ para faixa moderada e o mesmo valor percentual para estresse intenso totalizando $90 \%$ dos casos o que corrobora com a casuística de Lima ${ }^{11}$ onde os resultados da pesquisa indicaram que $93,33 \%$ dos trabalhadores de saúde estão com nível de estresse de moderado a intenso. Aqueles que atuam na comunidade têm maior dificuldade adaptativa aos fatores estressantes, uma vez que não só se expõem continuamente a problemas de naturezas diversas, mas são frequentemente surpreendidos por eles.

Tabela 3. Sinais e sintomas mais prevalentes do estresse ocupacional dos ACS da USF Águas Brancas, Ananindeua-PA

\begin{tabular}{lcc}
\hline \multicolumn{1}{c}{ VARIÁVEL } & ACHADOS/TOTAL & $\%$ \\
\hline FADIGA & $17 / 20$ & 85 \\
\hline DOR NOS MUSCULOS PESCOÇO E OMBRO & $17 / 20$ & 85 \\
\hline DOR DE CABEÇA & $16 / 20$ & 80 \\
\hline INDIGESTÃO & $16 / 20$ & 80 \\
\hline DOR LOMBAR & $16 / 20$ & 80 \\
\hline NERVOSISMO & $13 / 20$ & 65 \\
\hline IRRITABILIDADE & $12 / 20$ & 60 \\
\hline
\end{tabular}

Fonte: Protocolo de pesquisa.

Tabela 4. Avaliação do estresse ocupacional dos ACS da USF Águas Brancas, Ananindeua-PA

\begin{tabular}{lcc}
\hline \multicolumn{1}{c}{ VARIÁVEL } & TOTAL & \% \\
SEM ESTRESSE & 2 & 10 \\
\hline ESTRESSE MODERADO & 9 & 45 \\
\hline ESTRESSE INTENSO & 9 & 45 \\
\hline
\end{tabular}

Fonte: Protocolo de pesquisa. 


\section{Considerações finais}

O presente artigo mostrou em síntese os casos de estresse ocupacional sendo que $90 \%$ dos ACS apresentam estresse moderado a intenso com sinais e sintomas prevalentes como fadiga, dor nos músculos pescoço e ombro com $85 \%$ dos casos seguidas de dor de cabeça indigestão e dor lombar com $80 \%$. Sexo feminino, pardas, escolaridade ensino médio completo, faixa etária de 40- 49 anos, Em se tratando de fatores preceptores do estresse no trabalho a partir do inquérito ocupacional temos $70 \%$ que classificaram como média a satisfação com o trabalho, $75 \%$ consideram baixo o conforto no ambiente de trabalho, $40 \%$ afirmam que a temperatura é que mais causa incomoda quanto as condições do ambiente, $40 \%$ afirmaram que o volume de serviço é excessivo sazonal, quanto a lateralidade $70 \%$ são destros, $40 \%$ são estudantes e $25 \%$ realizam trabalhos extras. $15 \%$ sofreram acidente de trabalho, todos trabalham 8 h durante a semana e $25 \%$ trabalham nos fim de semana na informalidade

Essa situação serve de alerta para que medidas corretivas e preventivas possam ser tomadas a fim de evitarmos a queda de desempenho e o adoecimento desses trabalhadores o que sinaliza e mostra a importância de investimentos em políticas públicas voltadas a saúde do trabalhador.

\section{Referências}

1. Datti D. Mecanismos e prevenção do stress. Rio de Janeiro: Rosa dos Tempos; 1987.

2. Pereira, AMTB. Burnout: quando o trabalho ameaça o bem-estar do trabalhador. São Paulo: Casa do Psicólogo; 2008.

3. Maia LDG., Silva ND., Mendes PHC. Síndrome de Burnout em agentes comunitários de saúde: aspectos de sua formação e prática. Rev Bras Saúde Ocup.2011;36(123):93-102. http://dx.doi.org/10.1590/S0303-76572011000100009.

4. Santos IER. Estresse ocupacional em agentes comunitários de saúde da cidade de Aracaju Sergipe [dissertação]. Aracaju (SE): Universidade Tiradentes; 2011.

5. Brasil. Ministério da Saúde. Programa de Saúde da Família. Saúde da família: uma estratégia de organização dos serviços de saúde. Brasília (DF): Ministério da Saúde; 1994

6. Resende MC, Azevedo EGS, Lourenço LR, Faria LS, Alves NF, Farina NP, et al. Saúde mental e ansiedade em agentes comunitários que atuam em saúde da família em Uberlândia (MG, Brasil). Ciên Saúde Colet. 2011;16(4):2115-22. http://dx.doi.org/10.1590/S1413-81232011000400011. PMid:21584453.

7. Baccaro A. Vencendo o stress - como detectá-lo e superá-lo. Rio de Janeiro: Vozes; 1998.

8. Santos KT, Saliba NA, Moimaz SAS, Arcieri RM, Carvalho ML. Agente comunitário de saúde: perfil adequado a realidade do Programa Saúde da Família?. Ciên Saúde Colet. 2011;(Supl 1):16:1023-28. http://dx.doi.org/10.1590/S1413-81232011000700035.

9. Mota CM, Dosea GS, Nunes PS. Avaliação da presença da Síndrome de Burnout em Agentes Comunitários de Saúde no município de Aracaju, Sergipe, Brasil. Ciênc. Saúde Coletiva. 2014;19(12), 4719-26. http:// dx.doi.org/10.1590/1413-812320141912.02512013.

10. Cartotto MS, Câmara SG. Análise da produção científica sobre a Síndrome de Burnout no Brasil. Rev Psico. 2008;39(2):152-8.

11. LIMA JC. Stress ocupacional dos trabalhadores da saúde[monografia]. Criciúma (SC): Universidade do Extremo Sul Catarinense; 2010. http://docplayer.com.br/16600372-Stress-ocupacional-dos-trabalhadores-da-saude.html.

12. Glina DMR. Fatores de estresse no trabalho de operadores de centrais de atendimento telefônico de um banco em São Paulo. Rev Bras Méd Trab. 2003;1(1):31-9.

\section{Autor correspondente}

Cleverton Chaves dos Reis

Universidade do Estado do Pará - UEPA

Tv. Mauriti, 2095, Marco

CEP 66093-180, Belém, PA, Brasil

Tel.: (91) 99304-8102

E-mail: clevertoncreis@hotmail.com

\section{Informação sobre os autores}

CCR é médico de família e Comunidade, graduado pela Universidade do Estado do Pará (UEPA); especialização em Medicina do Trabalho; professor substituto da Universidade do Estado do Pará.

SAOM é médico de família e comunidade graduado pela Universidade federal do Pará; mestre em clínica de doenças tropicais pelo Núcleo de Medicina Tropical da Universidade Federal do Pará (UFPA); professor Assistente III da Universidade do Estado do Pará.

\section{Contribuição dos autores}

Todos os autores contribuíram igualmente para a realização do estudo.

Todos os autores leram e aprovaram a versão final submetida ao Pará Research Medical Journal. 